\title{
The role of attention in the enumeration of canonical patterns
}

\author{
Gordon Briggs $^{1}$ (D) . Christina Wasylyshyn ${ }^{1} \cdot$ Paul F. Bello $^{1}$
}

Published online: 9 March 2020

(C) This is a U.S. government work and its text is not subject to copyright protection in the United States; however, its text may be subject to foreign copyright protection 2020

\begin{abstract}
We report novel findings from experiments on the enumeration of canonical patterns under attentional load. While previous studies have shown that the process of enumerating randomized arrangements can be disrupted by attentional load, the effect of attentional load on canonical patterns has been unexplored. To investigate this case, we adapted a spatial dual-task paradigm previously used to study attentional disruption during the enumeration of randomized arrangements. We begin by replicating previous findings for randomized arrangements, with enumeration error increasing with cluster numerosity and attentional load. For dice patterns, enumeration error also increased under attentional load. However, contrary to findings from studies on single-task enumeration of dice patterns, we observed conflation of patterns with similar outlines. In subsequent experiments, we manipulated the spatial location of the enumeration task, placing the dot cluster in the center. With centrally located, canonical patterns that remained in the same location across trials, enumeration accuracy was more consistent with results from single-task studies. We hypothesize that participants may be using shape cues to inform guessing during enumeration tasks when unable to both localize and fully attend to target patterns.
\end{abstract}

Keywords Numerosity judgment $\cdot$ Enumeration $\cdot$ Attention $\cdot$ Pattern recognition $\cdot$ Canonical patterns

\section{Introduction}

Common arrangements of visual objects, often called canonical patterns, are enumerated more rapidly and more accurately under time constraints than randomly arranged ones (Mandler \& Shebo, 1982; Krajcsi et al., 2013). For example, patterns such as those found on six-sided dice are enumerated quickly (under 200-250 ms) and with little error (Wender \& Rothkegel, 2000; Jansen et al., 2014). As canonical patterns hold processing advantages over noncanonical patterns, and the enumeration of previously unfamiliar patterns can be improved with repeated exposure (Wolters et al., 1987), the ability to leverage a pattern-recognition process in numerosity judgment is uncontroversial.

Yet, what confers a processing advantage of canonical patterns over noncanonical patterns? Currently, little is understood about the limitations of the enumeration of

Gordon Briggs

gordon.briggs@nrl.navy.mil

1 Navy Center for Applied Research in Artificial Intelligence, U.S. Naval Research Laboratory, 4555 Overlook Ave SW, Washington, DC 20375 USA canonical patterns that could inform us as to its underlying processes. In contrast, the psychophysical limitations of the enumeration of randomly arranged visual objects have been more extensively explored. Beginning in the 19th-century, the enumeration of small quantities has been shown to be both rapid and accurate (Jevons, 1871). This phenomenon was later termed subitizing (Kaufman et al., 1949). While there was early debate regarding the size of the subitizing range, it is now generally considered to be 1-4 items for randomly arranged visual objects (Trick \& Pylyshyn, 1994). Within this range, there is only a small (40-100 $\mathrm{ms}$ ) reaction time increase per additional visual item (Trick, 1992) and enumeration is highly accurate even with brief presentation durations (e.g., 200-400 ms) (Mandler \& Shebo, 1982). Additionally, between 1-3 items, generally there is no significant effect of quantity on enumeration accuracy (Mandler \& Shebo, 1982). However, beyond the subitizing range, exact enumeration of additional items requires additional time, roughly $250-350$ milliseconds per item (Trick \& Pylyshyn, 1994). Consequently, enumeration accuracy decreases outside the subitizing range for shorter presentation durations.

The question of what processes underlie subitizing is still the subject of debate (Choo \& Franconeri, 2014). Given the strong effects of spatial configuration on enumeration 
performance, some propose that pattern recognition plays a key role in subitizing (Mandler \& Shebo, 1982; Wender \& Rothkegel, 2000; Krajcsi et al., 2013). Previous computational and mathematical models of enumeration by pattern recognition have not only proposed templatematching accounts where a particular spatial arrangement of visual items is mapped to a quantity, but also argue that the four-item subitizing range is an emergent phenomenon of the dramatically increased number of possible templates for higher quantities (Peterson \& Simon, 2000; Logan $\&$ Zbrodoff, 2003). Furthermore, Peterson and Simon (2000) propose that the increased reaction time per visual item could be accounted for by decreased activation in templates of higher numerosity, due in part to competition between more numerous possible templates. The processing advantage held by canonical patterns could also be explained by such an account, as templates corresponding to canonical patterns, by virtue of their more frequent prior exposure, would have higher initial activation than other competing templates.

However, these models of enumeration by pattern recognition leave open the question of how visual attention is deployed to encode the information necessary to perform template matching. Prior research on visual perception has implicated selective attention in constructing durative representations of visual objects (Rensink, 2000; Treisman \& Gelade, 1980). Additionally, it has been demonstrated that patterns of multiple objects can also be selectively attended to and classified as task-relevant or irrelevant during an enumeration task (Trick \& Enns, 1997). One possibility is that subitizing involves a single step of selective attention to a group of visual items, during which the entire group is encoded in visual memory. Another possibility is that groups of visual items are decomposed into individual items and/or subgroups, which are then sequentially attended to and encoded in visual memory. Consistent with the former view, early work in studying the neurophysiological traces of enumeration showed signs of shifts of selective visual attention during counting, but not subitizing (Sathian et al., 1999). In contrast, more recent behavioral and neurophysiological evidence suggests that shifts of selective attention may also occur during subitizing.

In several studies, subitizing of randomized arrangements has been shown to be disrupted under conditions of attentional load, with enumeration accuracy significantly decreasing between 1-2 or 2-3 items (Railo et al., 2008; Olivers \& Watson, 2008; Egeth et al., 2008; Vetter et al., 2008; Burr et al., 2010). Coupled with findings from neurophysiological experiments that show markers of shifts of selective attention during subitizing (Hyde \& Wood, 2011; Mazza \& Caramazza, 2015), this provides evidence against accounts of subitizing that posit visual encoding of an arrangement of objects under a single step of selective attention. Other experiments have also demonstrated that increased load on visual working memory disrupts subitizing performance (Piazza et al., 2011). This suggests that not only might attention be deployed sequentially, but that visual encoding may also occur in a multi-step, incremental fashion. If a group of visual items must be decomposed into individuals or subgroups, then it makes sense that fewer elements can be encoded when visual working memory is already taxed. In light of these studies, other recent models of subitizing have appealed to incremental perception through rapid, sequential attention to individual visual items to account for the exact enumeration of small quantities (Hyde \& Wood, 2011; Briggs et al., 2017).

Sequential, attentional selection leading to incremental visual encoding would suggest another possible explanation for the canonical pattern advantage. In contrast to randomized arrangements, certain canonical patterns may be able to be decomposed into fewer and more easily recognizable subpatterns (Wender \& Rothkegel, 2000). For example, the dice-face pattern of five dots can be recognized in two steps: recognizing the outer square of dots followed by the central dot. However, five randomly arranged dots could have a much wider range of less structured subgroupings. Previous experimental data provides some support for such an incremental-integration account. Whereas Mandler and Shebo (1982) report negligible reaction-time differences for canonical patterns up to five, other studies have shown statistically significant (if slight) reaction time increases for enumerating dice-pattern arrangements of more than four dots (Wender \& Rothkegel, 2000). Another study shows that when participants are given very brief $(<30 \mathrm{~ms})$ presentations of structured patterns, either arranged linearly or as vertices of regular polygons, enumeration accuracy suffers more when items are arranged linearly (Allen \& McGeorge, 2008), indicating that some outline-based conflation may be occurring. Allen and McGeorge (2011) also showed that expert participants (in their case, air traffic controllers) enumerate structured patterns more accurately than novices, but do so at a time cost. Therefore, the integration of information from all items in a canonical pattern may occur in multiple steps of selective attention. Additionally, if enumeration of canonical patterns is an incremental, multi-step process, the distribution of error when disrupted (either through limited viewing time or attentional load) could inform us what intermediate representations are being encoded by the visual system. For instance, the findings from Allen and McGeorge (2008) and Allen and McGeorge (2011) suggest that information from objects on the outlines of patterns may be encoded earlier than information from the internal ones.

In this paper, we present the results of dual-task experiments designed to examine the enumeration of 
canonical patterns under conditions of attentional load. In contrast to the findings in single-task studies of canonical pattern enumeration (e.g., Mandler and Shebo (1982), Ashkenazi et al. (2013), Jansen et al. (2014), and Bloechle et al. (2018)), we found that the process of enumerating canonical patterns was disrupted by attentional load. Furthermore, analysis of the distribution of responses under higher attentional load showed evidence of conflation between patterns with similar contours, providing support for an incremental account of enumeration by pattern recognition. Specifically, dice patterns of six or five dots were often reported as four, while dice patterns of three dots were often reported as two. In subsequent experiments, we manipulated the location of the enumeration task. With centrally located patterns that appeared in the same location during trials, enumeration accuracy was more consistent with results from single-task studies. We hypothesize that participants may be using shape cues to inform guessing during enumeration tasks when unable to both localize and fully attend to target patterns.

\section{Experiment 1: Randomized vs. dice patterns}

To investigate the enumeration of canonical patterns in the context of attentional constraints, we adapted the dualtask paradigm presented by Railo and colleagues (2008). In their study, participants had two potential tasks: (1) report which of the vertical or horizontal axes of a centrally located cross is longer and (2) report the number of dots randomly clustered in a quadrant outside the central cross (see Fig. 1). Initially, participants viewed and responded to a few trials in which only the cross appeared (task 1 only). Then in a critical trial, in which a peripheral dot cluster appeared for the first time (and participants were unaware of the enumeration task), they were asked whether or not they noticed anything other than the cross. If participants reported seeing other objects, they were then asked how many they saw. After this critical trial, participants then performed trials in two counterbalanced blocks on videos containing both a cross and a peripheral cluster of dots.
In one block, participants were asked to perform both task 1 and 2, and in the other, participants were asked only to enumerate (task 2 only). Thus, trials could be grouped into three attentional conditions vis-á-vis enumeration: (1) the inattention condition, consisting of the critical trial; (2) the divided attention condition; and (3) the full attention condition.

Using this dual-task paradigm, Railo and colleagues (2008) found that enumeration accuracy in the dividedattention condition was significantly lower than in the fullattention condition, and accuracy decreased significantly even within the subitizing range. How canonical forms would be enumerated in similar conditions would depend on the attentional processes underlying pattern recognition.

The aim of Experiment 1 was two-fold. First, we sought to replicate the results from Railo and colleagues (2008) for randomized, noncanonical arrangements using participants recruited on the Amazon Mechanical Turk platform (see Paolacci et al. (2010) for a review). The second aim of the study was to investigate the effects of attentional load on enumeration of canonical patterns. If a patterned group of dots can be rapidly encoded with a single instance of selective attention, we predict that numerosity would not significantly affect enumeration accuracy for participants given dice pattern arrangements. This hypothesis contrasts with the effects found for randomized arrangements by Railo and colleagues (2008), where both enumeration accuracy generally decreased as the number of peripheral dots increased, and decreased significantly for larger numbers in divided-attention trials relative to full-attention trials.

We replicated the task from Railo and colleagues (2008) with two different dot-arrangement conditions. One was a randomized arrangement condition, which served as a control and basis for replication. The second was a dice pattern condition, in which all peripheral clusters of dots were arranged canonically. The right side of Fig. 1 illustrates examples of randomized and dice patterns for each numerosity probed. Comparisons between attentional conditions were within subjects.

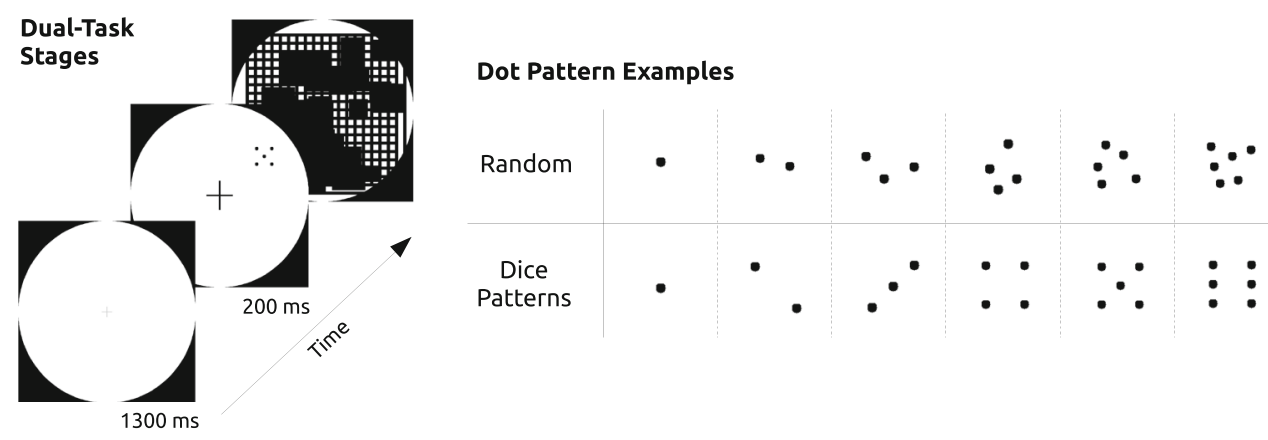

Fig. 1 Time course of dual-task videos (left). Examples of dot arrangements used in each pattern category (right) 

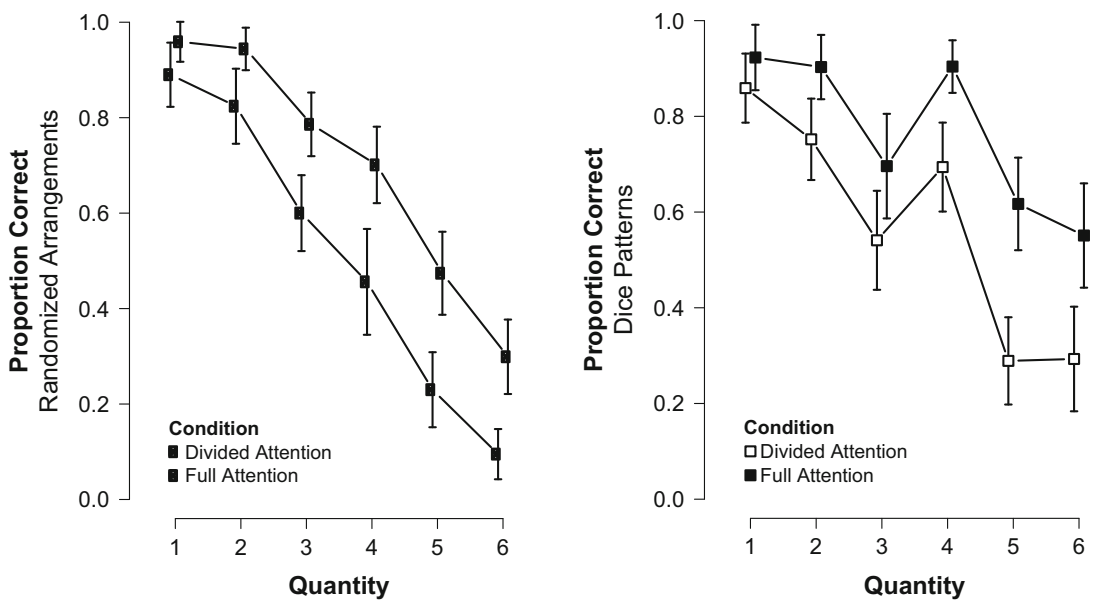

Fig. 2 (Experiment 1) Accuracy of numerosity judgment in divided- and full-attention conditions for randomized arrangements (left) and dice-pattern dot arrangements (right) presented for $200 \mathrm{~ms}$. Error bars indicate $95 \%$ confidence interval

\section{Method}

Participants Eighty-eight participants volunteered through the Amazon Mechanical Turk platform. Participants were almost evenly distributed between the randomized arrangement and dice pattern conditions, with 45 and 43 participants, respectively. The task was configured to be unavailable to users on mobile platforms to ensure appropriate viewing size of task videos. Self-reported demographic information indicates a mean age of 35.8 years old ( 50 male, 36 female, and 2 other participants; $S D=9.4$ ).

Stimuli The left side of Fig. 1 illustrates the time course of the dual task. First, a small fixation cross appears in the center of a circular viewing area for 1.3 seconds. The task stimulus consisting of the centrally located cross and the peripherally-located cluster of dots then appears for $200 \mathrm{~ms}$. A masker then appears and remains on the screen while each participant responds to the task questions. In each video, the dimensions of the viewing area and task objects were designed to replicate the stimuli from Experiment 2 from Railo and colleagues (2008) as closely as possible, given the inability to control the viewing hardware of each participant. All pixel dimensions were calculated based on an assumption of 100 pixels per inch (ppi) using the metrical information specified by Railo and colleagues (2008). Videos were $706 \times 706$ pixels in size. Dots were 13 pixels in diameter. Dice patterns were contained in $60 \times 60$ pixel regions, while randomized patterns were contained in $70 \times 70$ pixel regions. Though, because they were randomly generated, these arrangements rarely had a width or height of 70 pixels. The allowable region for the randomized arrangements was increased to allow for dot patterns of 4 6 to have continuous properties (e.g., convex hull length, additive area, etc.) closer to their dice pattern counterparts. ${ }^{1}$ For dice patterns with vertical or horizontal asymmetry (i.e., two and three), both mirrored variants were generated. The centrally-located crosses during divided-attention trials were either $104 \times 113$ or $113 \times 104$ pixels in diameter.

Procedure After completing six cross-only trials and the inattention trial, participants then completed two blocks corresponding to each attentional condition. Each block consisted of 22 trials. For the full-attention block, instructions indicated that participants would be asked about the number of peripheral dots and not the cross dimensions, whereas the divided-attention instructions indicated that participants would first be asked about the cross dimensions. The order of these blocks was counterbalanced; half of the participants received the full attention block first, while the other half received the divided-attention block first. Each peripheral-cluster numerosity was presented within each block between three to six times, ${ }^{2}$ such that there were 22 trials per block. Some variability in the number of trials was introduced to attempt to mitigate participants informing guesses based on what had been seen already, particularly in the later block. The order and frequency of presentation for each numerosity were randomized independently for

\footnotetext{
$\overline{{ }^{1} \text { Additionally, }}$ our analysis does not involve direct between-subjects comparisons between participants observing dice patterns and participants observing randomized patterns.

${ }^{2}$ The minimum number of trials in our experiment is greater than the minimum number of trials for each quantity presented in the original study from Railo et al. (2008), in which a specific quantity was only guaranteed to be presented at least once. It is also similar to other studies involving enumeration by pattern recognition (Ashkenazi et al., 2013).
} 
each participant. The quadrant of the viewing area that the peripheral cluster would appear in was also randomized, appearing with equal probability in each quadrant. When participants were asked to report perceived numerosity of the peripheral dots, they were given the option to select values ranging from zero to seven in a drop-down menu. Participants were also asked how confident they were in their responses $(1=$ very unsure to $5=$ very sure $)$.

\section{Results}

\section{Cross task accuracy}

As with the original study (Railo et al., 2008), accuracy on the cross task in the divided-attention trials for randomized dot-arrangements was above chance (69.7\% correct). A onesample $t$-test showed that this was significant $(t(42)=$ $6.48, p<.001)$ and was not correlated with enumeration accuracy (Spearman's rho, $\rho=-.195, p=.210$ ), indicating that participants were dividing attention between the cross and enumeration tasks. In the dice-pattern condition, accuracy on the cross task in the divided-attention trials $(71.9 \%$ correct) was also found to be significantly above chance $(t(44)=9.18, p<.001)$ and not correlated with enumeration accuracy (Spearman's rho, $\rho=-.126$, $p=.411)$.

\section{Enumeration accuracy}

Enumeration accuracy in each condition is graphed in Fig. 2. Data were analyzed with a mixed ANOVA with within-subject factors of attentional condition and cluster numerosity and with a between-subjects factor of cluster arrangement. When violations of sphericity were detected, the Greenhouse-Geisser correction was applied. Significant within-subjects effects of attentional load, $F(1,86)=106.56, p<.001$, and cluster numerosity, $F(4.03,346.96)=157.96, p<.001$, were found. Interaction effects were found between numerosity and arrangement, $F(4.03,346.96)=13.14, p<.001$, and numerosity and attentional load, $F(4.45,382.52)=5.23$, $p<.001$.

Randomized Arrangements In the inattention trial, only 11 out of 43 participants $(26 \%)$ reported seeing the peripheral dot cluster. This was lower that the $61 \%$ reported by Railo and colleagues (2008), and given the resulting limited amount of data, we did not conduct post-hoc analyses of enumeration accuracy in the inattention trials. However, analysis of the divided- and full-attention data from participants in the randomized-arrangement condition showed successful replication of the key effects found by Railo and colleagues (2008). Enumeration accuracy was found to monotonically decrease as cluster numerosity increased in both divided- and full-attention conditions. We conducted post-hoc testing comparing the relative accuracy of adjacent numerosities and between the same numerosity between attentional conditions. Because of these multiple comparisons, we applied the Bonferroni correction to adjust the acceptance criterion to $\alpha=.0167$. Paired-samples $t$ tests showed significant decreases in enumeration accuracy during divided-attention trials between numerosities 2-3 $(t(42)=4.77, p<.001$, Cohen's $d=.728) ; 4-5(t(42)=$ $3.94, p<.001$, Cohen's $d=.601)$; and 5-6 $(t(42)=$ 2.94, $p=.005$, Cohen's $d=.449)$. Significant decreases in enumeration accuracy were found during full-attention trials between numerosities $2-3(t(42)=4.23, p<.001$, Cohen's $d=.646)$; 4-5 ( $t(42)=4.25, p<.001$, Cohen's $d=.649)$; and 5-6 $(t(42)=3.12, p=.003$, Cohen's $d=.476)$. Finally, enumeration accuracy was significantly lower in divided attention trials versus full attention trials, with small effects found for numerosities 1-2 $(t(42)>$ $2.65, p<.011$, Cohen's $d>.405)$ and medium to large effects found for numerosities $3-6(t(42)>3.62, p<.001$, Cohen's $d>.553$ ).

Dice Patterns As with randomized arrangements, few participants in the dice-pattern condition also noticed the peripheral dot cluster in the inattention trial. Only 10 out of 45 participants (22\%) reported seeing the peripheral dot cluster, so as with randomized arrangements, we did not conduct a post-hoc analysis of enumeration accuracy in the inattention trials. In the divided- and full-attention trials, contrary to our original predictions, we found that enumeration accuracy of dice patterns was affected by both numerosity and attentional condition. Paired-samples $t$ tests showed significant decreases in enumeration accuracy during divided-attention trials between numerosities 2-3 $(t(44)=3.38, p=.002$, Cohen's $d=.503)$ and $4-5$ $(t(44)=6.79, p<.001$, Cohen's $d=1.01)$. However, enumeration accuracy increased between numerosities 3$4(t(44)=-2.51, p=.016$, Cohen's $d=-.374)$. Enumeration accuracy during full-attention trials was found to follow a similar pattern with accuracy decreasing between $2-3(t(44)=4.03, p<.001$, Cohen's $d=.601)$, increasing between $3-4(t(44)=-3.85, p<.001$, Cohen's $d=-.574)$, and decreasing again between $4-5(t(44)=$ $6.18, p<.001$, Cohen's $d=.921)$. Enumeration accuracy was lower in divided attention trials versus full attention trials, with significant decreases found for numerosities 2 and 4-5 ( $t(44)>3.51, p \leq .001$, Cohen's $d>.524)$.

In addition to countering our original predictions, these results are sharply different from those found in single task studies of enumerating canonical patterns. For instance, 
Ashkenazi and colleagues (2013) showed enumeration accuracy at ceiling (>95\%) for dice patterns with 200 ms presentations for their neurotypical control population. There are a few differences between our task and theirs that may be a source of such divergent results, including increased task difficulty (as even in the full-attention trials, it is unknown which quadrant the pattern will appear). Another key difference between our stimuli and those used by Ashkenazi and colleagues (2013) is that our critical task phase is immediately followed by a masker, whereas theirs is followed by $500 \mathrm{~ms}$ of a blank screen with no subsequent masker. This raises the possibility that in the task by Ashkenazi and colleagues (2013), participants were able to complete their perception of canonical patterns using iconic memory traces (Coltheart, 1980). These results are, however, consistent with the findings from Allen and McGeorge (2008), who found evidence for the conflation of linearly arranged patterns. It is worth noting that Allen and McGeorge (2008) used patterns located in the center of a participant's field of view, which would argue against this effect being simply an artifact of eccentricity.

\section{Enumeration confidence}

Enumeration confidence-ratings for all experiments are plotted in Fig. 7 in Appendix A. Confidence data for Experiment 1 were analyzed with a mixed ANOVA. The within-subjects factors were attentional condition and cluster numerosity and the between-subject factor was cluster arrangement. Significant within-subjects effects of attentional load, $F(1,86)=79.032, p<.001$, and cluster numerosity, $F(3.40,292.0)=88.93, p<.001$, were found. A significant interaction effect was found between numerosity and arrangement, $F(3.40,292.0)=4.773, p=$ .002 .

Randomized Arrangements As with enumeration accuracy, participants' reported ratings of confidence in their numerosity response monotonically decreased as the cluster numerosity increased. Paired samples $t$-tests indicated significant decreases in confidence ratings in divided-attention trials between numerosities $1-2(t(42)=4.49, p<.001$, Cohen's $d=.685) ; 2-3(t(42)=4.25, p<.001$, Cohen's $d=.648)$; and 3-4 $(t(42)=2.51, p=.016$, Cohen's $d=.382$ ). This decrease in response confidence within the subitizing range also was observed in full-attention trials with significant decreases found between $1-2(t(42)=$ $3.72, p<.001$, Cohen's $d=.567) ; 2-3(t(42)=3.85$, $p<.001$, Cohen's $d=.587)$; and 3-4 $(t(42)=, p=.006$, Cohen's $d=.438$ ). Finally, participants were less confident in divided-attention trials versus full-attention trials for all numerosities with medium to large effects detected for numerosities $1-5(t(42)>4.19, p<.001$, Cohen's $d>$.639) and a smaller effect detected for $6(t(42)=3.02$, $p=.004$, Cohen's $d=.46$ ). The observed decrease in confidence is consistent with accounts of subitizing based on rapid sequential focus of attention (e.g., Railo et al. (2008), Hyde and Wood (2011), and Briggs et al. (2017)). Given a limited time to enumerate, it becomes less likely the participant has fully attended to all items to exactly enumerate or attended to a subset of items to substantially refine an estimate.

Dice Patterns As with randomized arrangments, enumeration confidence for participants in the dice pattern condition also tended to decrease as numerosity increased. Pairedsamples $t$-tests indicated significant decreases from 1-2 $(t(44)=2.68, p=.010$, Cohen's $d=.400)$ and from $2-3(t(44)=2.58, p=.013$, Cohen's $d=.385)$. In fullattention trials, confidence also significantly decreased from $1-2(t(44)=2.94, p=.005$, Cohen's $d=.438)$ and from $2-3(t(44)=3.90, p<.001$, Cohen's $d=.582)$. However, unlike in the divided-attention trials, enumeration confidence during full-attention trials increased from 3-4, though this was not significant, and decreased again from $4-5(t(44)=3.76, p<.001$, Cohen's $d=.560)$. Partipicants were significantly more confident in enumeration responses in full-attention trials than divided-attention trials in all numerosities $(t(44)>3.86, p<.001$, Cohen's $d>$.578). This finding provides further evidence that unlike in previous single-task studies, enumeration by pattern recognition is disrupted by attentional load. The fact that enumeration confidence was lower for numerosity 4 6 in divided attention trials and 5-6 in full attention trials indicates that participants were also not falsely confident in their numerosity judgments. This could indicate that they knew that the incremental encoding of cluster of dots had not been fully completed.

\section{Response distribution}

Not only did the data show attentional effects on enumeration accuracy for dice-pattern arrangements, but the pattern of responses was also consistent with participants relying on shape/outline information to guide numerosity judgments, especially in the case of high attentional load. We present confusion matrices of the numerosity responses given by participants in Experiment 1 in Fig. 3 to further illustrate this pattern of response.

In the divided-attention trials for dice patterns, not only is three confused with two, but five and six appear to be frequently confused with four. This pattern of conflation can also be found in the full-attention condition, despite the correct quantity response being selected more frequently. For trials with three dots in the full-attention, 

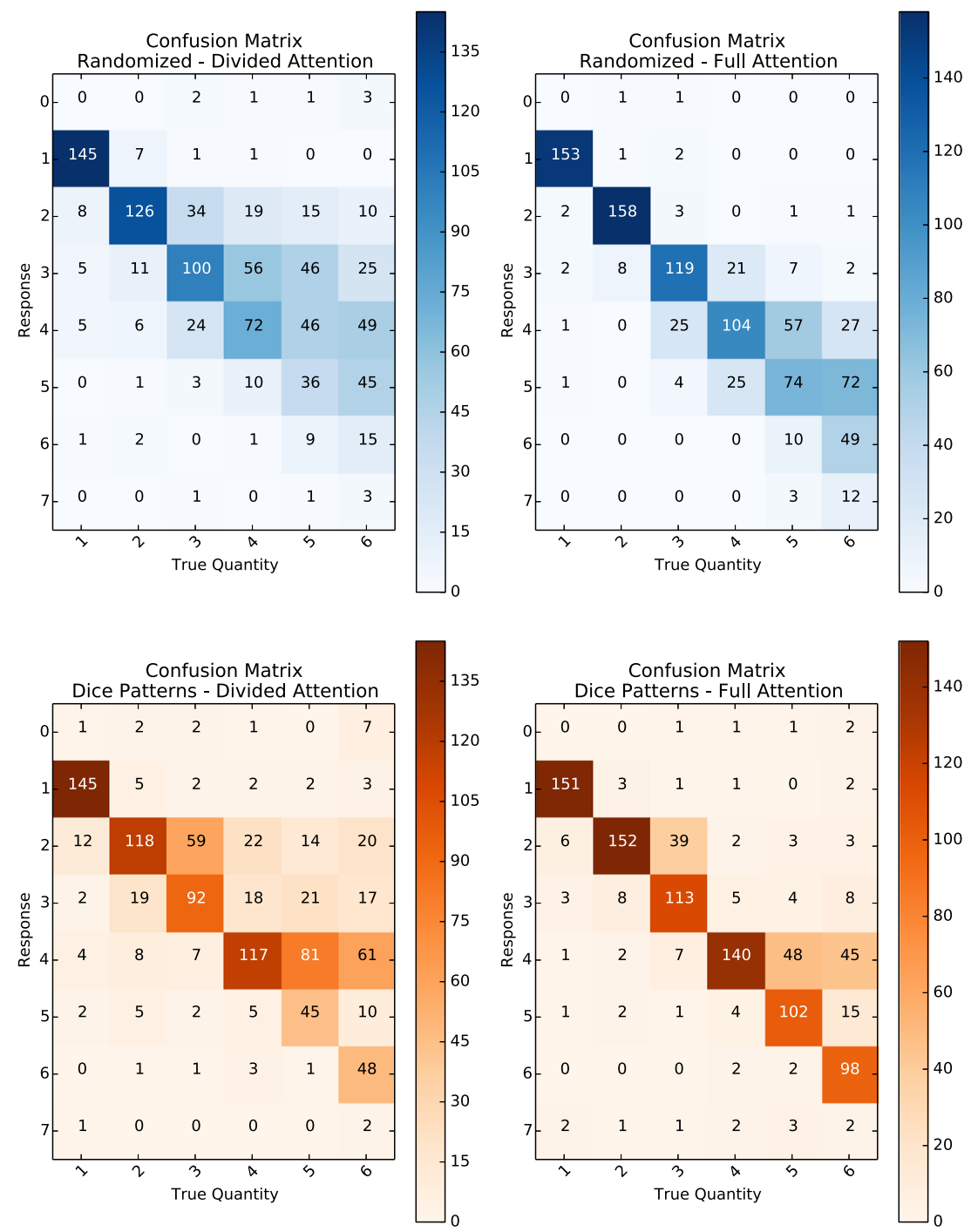

Fig. 3 Confusion matrices showing response distribution for each arrangement and attention condition in Experiment 1

dice-pattern condition, two was the second most frequent response. Likewise, for trials with five and six dots, four was the second most frequent response. The distribution of responses is also revealing. The pattern of response for randomized arrangements is more consistent with the distributions evinced by numerical estimation (e.g., Whalen et al., (1999)), specifically a continuous, gaussian-like distribution centered around a number close to (or equal to) the true numerosity. However, the distribution of responses for trials with six dots in the dice pattern condition is less gaussian-like, with four and six being reported significantly more than five.

One possible interpretation of this observed pattern of response distribution is that during enumeration by pattern recognition, participants first have access to shape/outline cues, then attempting to disambiguate similar patterns by subsequently focusing on uniquely identifying subregions. However, with the limited amount of time to enumerate, especially in the divided-attention trials, this second step is not achieved. An explanation involving initial detection and encoding of visual items along the outline of clusters is consistent with findings by Jewell (2007), who demonstrated that participants who briefly view (40$240 \mathrm{~ms}$ ) randomized dot clusters appear to exhibit change blindness toward missing/added dots in the interior of the cluster, whereas change to dots along the contour of the clusters were detected at above chance levels. This explanation would also be consistent with previous findings showing that the number of concentrically embedded shapes cannot be subitized (Trick \& Pylyshyn, 1994). Rather, sequential attention must be deployed to each layer of the concentrically embedded visual objects. While dots on the outline of a pattern do not form a continuous shape, it could be the case that they are represented as such during enumeration. Inner dots would then need additional time to enumerate. 
Previous studies have shown that similar behavior of outline conflation can occur when patterns are centrally located (Allen \& McGeorge 2008, 2011), suggesting that the peripheral location itself is not a cause of this effect. However, enumeration task location could still have an effect for other reasons. If tasks are attended to sequentially, the time cost of switching attention between tasks in a dualtask setting could cause reduced performance in the second task. Likewise, randomized stimuli locations would also potentially make the task more time consuming.

\section{Experiment 2: Controlling for task location}

In Experiment 2, we sought to evaluate the extent to which the observed disruption to canonical pattern enumeration was due to the location of the presented patterns. Because the location of peripheral stimuli varies between trials, one possibility is that the time it takes to first localize the peripheral stimuli reduces the amount of time available to subsequently attend to and perform the associated peripheral task. To investigate this possibility, in Experiment 2, we switched the locations of the cross dimension and enumeration tasks (i.e., the cross task became the peripheral task and the enumeration task became the central task). Furthermore, we modified the design of the experiment to include a within-subjects comparison of cluster arrangement, resulting in three conditions: a cross-task only full-attention condition, a randomized arrangement divided-attention condition, and a dice pattern divided-attention condition.

In an initial study swapping the locations of the cross dimension and enumeration tasks, we used the same cross dimension as Experiment 1. However, accuracy on the cross dimension task was at chance for many participants in the divided attention trials, indicating that they were unable to successfully attend to both tasks. Therefore, in Experiment 2, we made the peripheral cross task easier to demonstrate that participants were actually attending to both tasks in divided-attention trials. We ran a pilot study, detailed in Appendix B, to determine new dimensions for the presented task cross, selecting the dimensions with the highest task accuracy.

\section{Method}

Participants Forty-two participants volunteered through the Amazon Mechanical Turk platform. As with the previous experiments, the task was configured to be unavailable to users on mobile platforms to ensure appropriate viewing size of task videos. Self-reported demographic information indicates a mean age of 36.6 years old (21 male and 21 female participants; $S D=11.0$ ).
Stimuli During the critical stimulus interval, the dot arrangement appeared in the center of the screen, while the cross appeared in a random quadrant. Aside from this change, the stimuli characteristics were the same as in Experiment 1, except for the modified task cross dimensions. Based on the results of the cross task pilot study, the peripheral cross dimensions were changed to $93 \times 127$ pixels (such that the longer arm is roughly 1.37 times the length of the shorter arm).

Procedure Participants completed three blocks of trials corresponding to each attentional and arrangement condition. The full-attention cross-task block consisted of 12 trials. Divided-attention blocks consisted of 20 trials. For the full-attention block, instructions indicated that participants would be asked about the relative cross dimensions and not the dot cluster numerosity, whereas the divided attention instructions indicated that participants would be asked about both the cross dimensions and cluster numerosity. The order of these blocks was counterbalanced. For divided-attention blocks, cluster numerosities of one or two were presented either two or three times randomly, for a total of five trials for numerosities one and two. ${ }^{3}$ Numerosities of three to six were presented three to six times randomly, for a total of fifteen trials. As in Experiment 1, the order and frequency of presentation for each numerosity were randomized independently for each participant. The quadrant of the viewing area that the peripheral cross would appear in was also randomized (appearing with equal probability in each quadrant). As in Experiment 1, when participants were asked to report perceived numerosity of the central dot cluster, they were given the option to select values ranging from zero to seven in a drop-down menu. Participants were also asked how confident they were in their responses $(1=$ very unsure to $5=$ very sure).

\section{Results}

\section{Enumeration accuracy}

Enumeration accuracy in Experiment 2 for each condition is graphed in Fig. 4. Data were analyzed with a repeated measures ANOVA with within-subject factors of cluster arrangement and cluster numerosity. Significant effects

\footnotetext{
${ }^{3}$ Numerosities of one and two were presented for fewer trials on average as the enumeration literature consistenly demonstrates that enumeration accuracy of one or two items is at ceiling (e.g., Mandler and Shebo (1982) and Railo et al. (2008). Therefore, we would not expect any significant effects of decreased practice on the enumeration performance for these numerosities.
} 

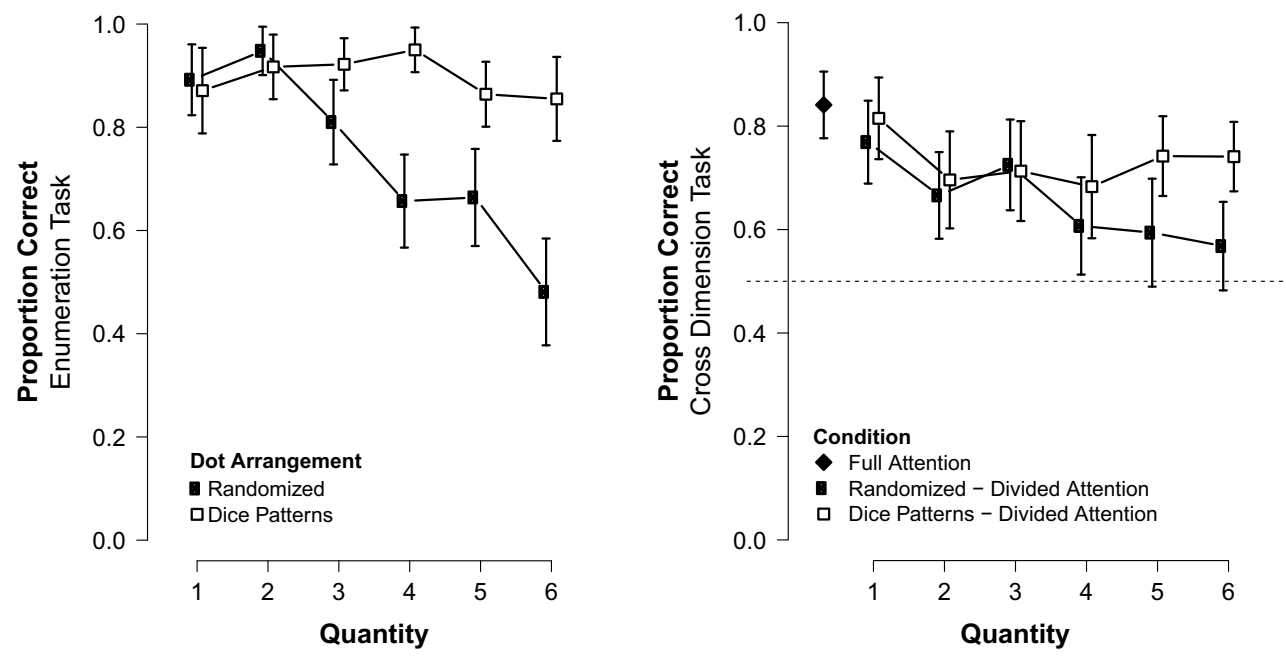

Fig. 4 Accuracy of numerosity judgments in Experiment 1 for randomized-arrangements and dice patterns (left). Accuracy of cross task responses in Experiment 2 given arrangement condition in the enumeration task (right). Error bars indicate $95 \%$ confidence intervals

of cluster arrangement, $F(1,41)=63.99, p<.001$, and cluster numerosity, $F(5,205)=16.77, p<.001$, were found. An interaction effect was also found between cluster arrangement and numerosity, $F(3.88,158.84)=$ $12.81, p<.001$. For post-hoc testing, a Bonferroni correction was applied as in Experiment 1. Accuracy for dice patterns remained high, with no significant changes in accuracy detected between numerosities. For randomized arrangements, enumeration accuracy generally decreased as numerosity increased. The first significant drop in enumeration accuracy for randomized arrangements occurred between numerosities $2-3(t(41)=3.24$, $p=.002$, Cohen's $d=.500$ ). Significant decreases in accuracy were also detected between numerosities 3$4(t(41)=2.61, p=.013$, Cohen's $d=.402)$ and 5-6 $(t(41)=3.48, p=.001$, Cohen's $d=$ .537). For higher numerosities (specifically four through six), dice patterns were enumerated more accurately than randomized arrangements $(t(41)>3.98, p<.001$, Cohen's $d>$.614). These results are more consistent with single-task enumeration studies where: (1) enumeration accuracy of dice patterns remains high for all quantities, and (2) enumeration accuracy of dice patterns is higher than enumeration accuracy of randomized arrangements outside the subitizing range.

\section{Enumeration confidence}

Enumeration confidence data were analyzed with a repeated measures ANOVA. Significant within-subject effects of cluster arrangement, $F(1,41)=36.86, p<.001$, and cluster numerosity, $F(3.62,148.21)=19.31, p<.001$, were found. An interaction effect was also found between cluster arrangement and numerosity, $F(4.37,179.04)=$ 20.36, $p<.001$.

As with enumeration accuracy, mean enumeration confidence for randomized arrangements decreased as numerosity increased. Paired-sample $t$-tests indicated a significant decrease between numerosities $2-3(t(41)=$ $3.11, p=.003$, Cohen's $d=.480), 3-4(t(41)=4.24$, $p<.001$, Cohen's $d=.655)$ and 5-6 $(t(41)=3.35$, $p=.002$, Cohen's $d=.516$ ). No significant differences in confidence ratings between numerosities were found for dice patterns, with confidence remaining high across numerosities. Confidence was found to be significantly lower for randomized arrangements than dice patterns for numerosities four through six $(t(41)>5.65, p<.001$, Cohen's $d>.871)$. These data are consistent with the notion that exact enumeration of randomized arrangements requires sequential attention. Additionally, these data are consistent with the notion that enumeration of randomized arrangements may require more attention to fully encode.

\section{Cross task accuracy}

A plot of cross task accuracy in Experiment 2 is found in Fig. 4. A one-sample $t$-test showed that participants performed significantly above chance $(84.1 \%)$ in fullattention (cross-task only) trials, $t(41)=10.4, p<$ .001 . The divided-attention trial data were analyzed with a repeated measures ANOVA with within-subject factors of cluster arrangement and cluster numerosity. A significant effect of cluster arrangement was found, $F(1,41)=$ $12.70, p<.001$. One-sample $t$-tests indicated that for all numerosities in divided attention, dice pattern trials, cross task accuracy was significantly above chance $(t(41)>$ 
3.60, $p<.001$, Cohen's $d>.556$ ). One-sample $t$ tests indicated that for numerosities between one and four in divided-attention, randomized arrangement trials, cross task accuracy was significantly above chance $(t(41)>$ 2.23, $p<.031, d>.344)$. Given this data, unlike in Experiment 2, it appears that participants were able to successfully complete both tasks. Unsurprisingly, the most difficult trials were the randomized arrangement trials with cluster numerosities outside the subitizing range.

\section{Response distribution}

We present confusion matrices of the numerosity responses given by participants in Experiment 2 in Fig. 5, which further illustrate the reduction of error and pattern conflation relative to Experiment 1. Of note, however, is that pattern conflation still appears to be present for the dice pattern of six: the majority of incorrect responses is still four.

\section{General discussion}

The results from the two experiments demonstrate that the enumeration of canonical patterns, like the enumeration of randomized arrangements, requires attention. Experiment 1 demonstrates that the enumeration of canonical patterns can be disrupted by attention load, and that this increased attentional load results in the conflation of patterns with similar contours. However, Experiment 2 showed that when the cluster to be enumerated is centered and consistently located across trials, enumeration performance of canonical patterns is more consistent with results from single-task studies. To account for these findings, we consider how attention is deployed during our tasks.

Evidence from electrophysiological studies of enumeration suggests that the transition in enumeration from spatial attention to a region of pre-individuated visual items and attention to individuated visual items occurs shortly preceding $200 \mathrm{~ms}$ (Mazza \& Caramazza, 2015). This aligns with the results from our experiments. Attention to a region of pre-individuated visual items may be sufficient to extract cues regarding the outline/shape of the cluster of items, but disambiguation of canonical patterns with similar outlines/shapes may require attention to specific, individuated items. The $200 \mathrm{~ms}$ presentation time found in Experiment 1 was likely insufficient, given the need to also perceive which quadrant the peripheral dot pattern appeared in (and perform the cross task in the divided-attention trials), to allow many participants to reach the individuation phase of numerical processing. The fact that the dot clusters in Experiment 2 were centrally-located and in the same location across trials may have enabled participants to devote more time to enumeration.

We illustrate this explanation in Fig. 6, which depicts multiple stages in the deployment of spatial attention in order to complete both the central cross task and peripheral enumeration task in Experiment 1. Spatial attention is first directed at the central cross (step 1). Next spatial attention is spread across the display to detect the peripheral dot cluster (step 2). Once the peripheral dot cluster is detected and localized, spatial attention is directed to its location (step 3) after which a pattern recognition process commences (step 4). At this point, multiple possible accounts exist.

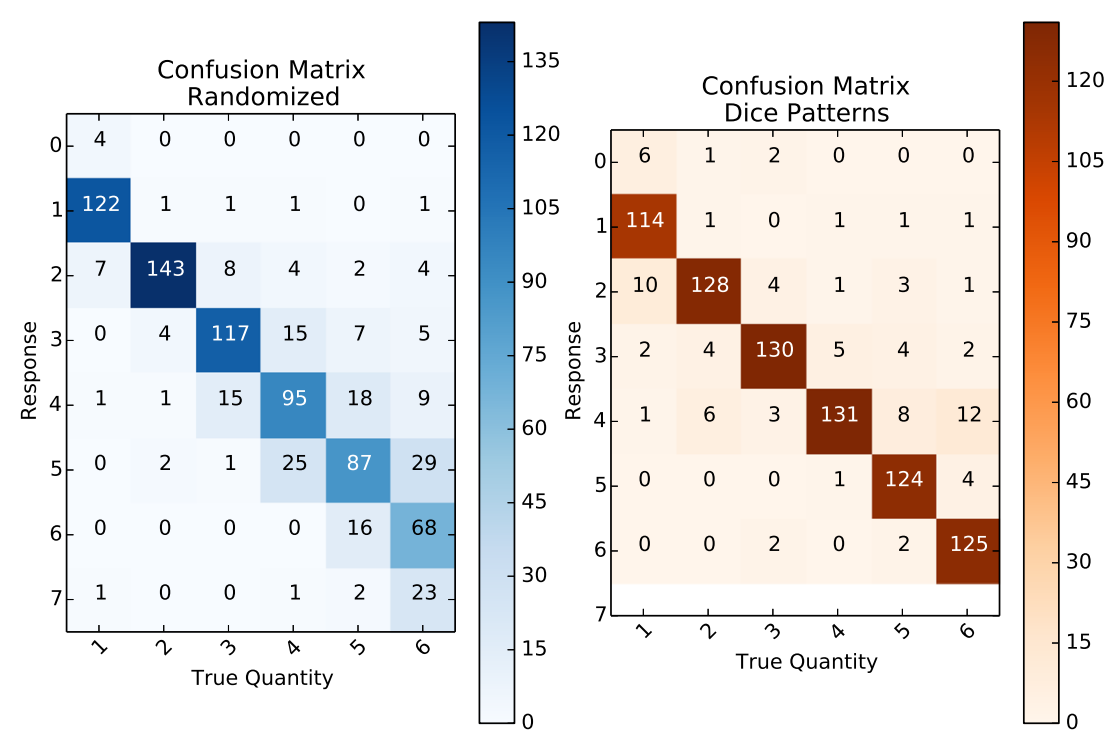

Fig. 5 Confusion matrices showing response distribution in Experiment 2 for each arrangement condition in divided attention trials 


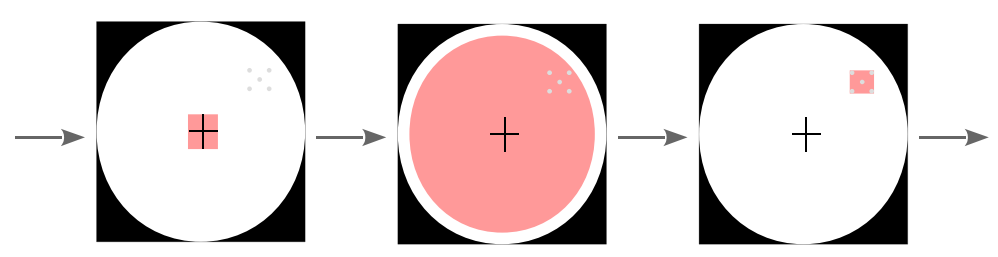

(1)

(2)

(3)

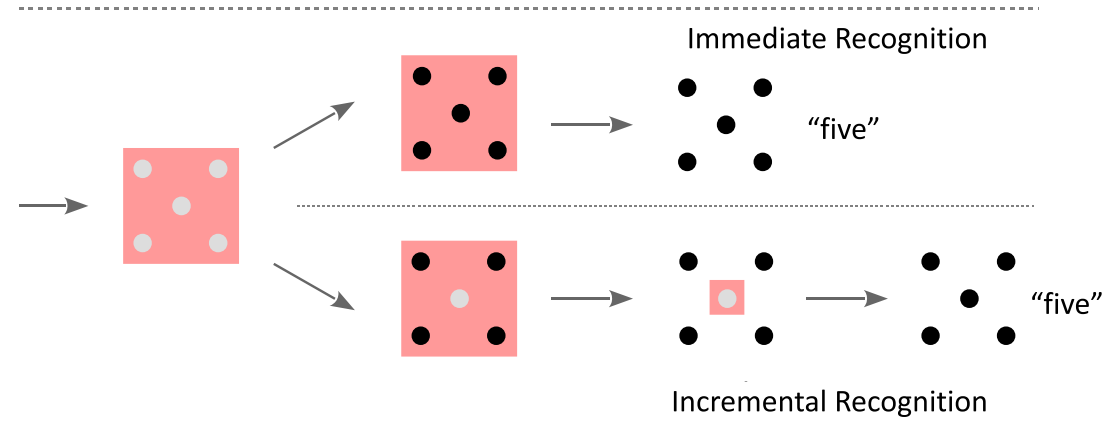

(3)

(4)

(5)

(6)

Fig. 6 Depiction of the hypothesized deployment of spatial attention the dual task from Experiment 1 (top) and different possible accounts of the recognition of canonical patterns (bottom). Attended areas highlighted by the red colored region. Greyed items indicate visual items that have not yet been processed and encoded in memory

Under the simultaneous-integration account of pattern recognition, the canonical pattern is then perceived and recognized (step 5, top). Under an incremental-integration account, a representation of the pattern must be constructed over multiple stages of attentional deployment (steps 56 , bottom). Yet another possible account of enumeration by pattern recognition involves two parallel processes, one rapid outline-detection process and one slower process that integration information from all visual items.

The results of Experiment 1 can be explained by disruption between steps 3-4 (and in the case of incremental or multiple process accounts, 4-5). If the stimulus interval ends and the masker appears in between these steps, then shape/outline information could still be derived and used to inform a guess regarding numerosity, leading to potential pattern conflation and lower enumeration confidence. Worse enumeration accuracy, confidence, and more pattern conflation in divided-attention trials relative to full-attention trials can be explained by the possibility that participants in full-attention trials would be starting at step 2, rather than step 1, leading to more potential time to complete the enumeration task. The central and consistent location of the enumeration task stimuli would effectively allow participants in Experiment 2 to start at step 3, allowing for even more time to fully process the canonical patterns, resulting in high enumeration accuracy and confidence across numerosities and little pattern conflation. This explanation is also consistent with the findings of pattern conflation for centrally located arrangements by Allen and McGeorge (2008) as patterns in those experiments were displayed for only $17-53 \mathrm{~ms}$. This suggests that even when patterns do not need to be detected and localized, the pattern recognition process can still be disrupted during extremely short presentation durations.

Likewise, this explanation also accounts for the results for randomized arrangements in our experiments. As both behavioral (e.g., Railo et al., (2008) and Burr et al. (2010)) and neurophysiological (Hyde \& Wood, 2011; Mazza \& Caramazza, 2015) studies lend increasing support to the role of sequential deployment of attention in the general enumeration of noncanonical arrangements of visual items, we would expect the enumeration in the randomizedarrangement trials to be interrupted with more frequency as numerosity increased. Not only did we find this in Experiment 1, which replicated prior findings from Railo et al. (2008), but evidence of this interruption was also found in the enumeration accuracy and confidence in Experiment 2, as well. In Experiment 2, when there was evidence that participants were attending to both tasks, the first significant decline in enumeration accuracy and confidence occurred within the subitizing range between 2-3. Furthermore, in Experiment 2, cross task accuracy 
during randomized arrangement trials declined to near chance levels for larger numerosities as well, consistent with participants being interrupted before being able to sufficiently attend to the secondary peripheral cross task.

In this paper, we have presented novel findings that demonstrate conditions during which enumeration of canonical patterns is and is not disrupted. The enumeration of canonical patterns, like the enumeration of randomized arrangements, requires attention. Under time constraints, when canonical patterns must first be detected and localized, the process of enumeration is interrupted. Enumeration responses, in these cases appear to be based on incomplete information based on the shape/outline of the target cluster. However, the enumeration of canonical patterns is less susceptible to interruption than the enumeration of randomized patterns. Some open questions still remain, such as whether or not the process of enumerating by pattern recognition can be interrupted after spatial attention is deployed to the target cluster of visual items. One possibility is that to fully recognize patterns, there is a need to deploy attention sequentially: first to establish a set of possible responses consistent with the gist of the arrangement, then to focus on subregions that would disambiguate between the multiple possibilities. One of the limitations of the present work is that dice patterns, even under this model, would require minimal additional deployments of attention to disambiguate patterns. However, more complex patterns might require more complex deployments of attention to disambiguate similar patterns. For instance, consider the dice pattern of four dots. Now consider the complete set of dot patterns of numbers 4-9 that would result in filling in the remaining dot positions in the $3 \times 3$ grid. Unlike with the dice patterns of 4-6, more positions within the pattern would require examination to differentiate it from other patterns. Would the enumeration of these patterns exhibit a distribution of error more akin to randomized arrangements? We leave the investigation of this possibility for future work.

Open Practices Statement Data and material from the experiments reported here will be made available on a Open Science Framework project site (https://osf.io/mx5ft/) pending institutional publication release approval. None of the experiments was preregistered.

Acknowledgments We are grateful to Kevin Zish, Kalyan Gupta, and the Knexus Research Corporation for their help in conducting the experiments and Andrew Lovett and Will Bridewell for their discussions on this project. We would like to thank Henry Railo for assistance in replicating his original study. Additionally, we would like to thank Attila Krajsci and other previous reviewers for helpful feedback. This work was supported by a NRC Research Associateship award to GB, and AFOSR MIPR grant \#F4FGA07074G001 and ONR grant \#N0001416WX00762, both awarded to PB. The views expressed in this paper are solely those of the authors and should not be taken to reflect any official policy or position of the United States Government or the Department of Defense.

\section{Appendix: A Enumeration confidence graphs}
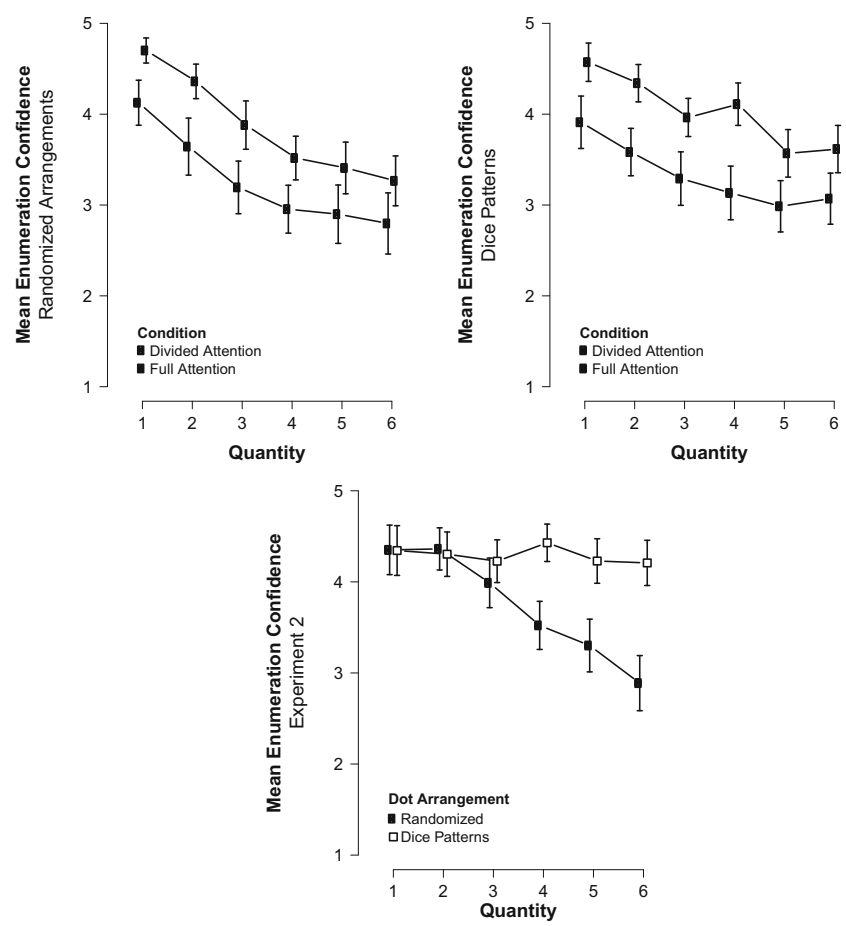

Fig. 7 Enumeration confidence in Experiment 1 during divided and full-attention trials for randomized arrangements (top left) and dice pattern dot arrangements (top right). Enumeration confidence in divided-attention trials for randomized arrangements and dice patterns in Experiment 2 (bottom). Error bars indicate 95\% confidence interval

\section{Appendix: B Cross task pilot study}

Fifteen participants volunteered on Amazon Mechanical Turk to engage in a pilot study to evaluate the difficulty of the peripheral cross task for varying cross dimensions. Four potential cross dimensions were tested. Eight videos for each set of dimension were created: four wider than tall crosses in each quadrant and four taller than wide crosses in each quadrant. Participants were given twenty trials, with five randomly selected videos for each cross dimension. The mean enumeration accuracy for each cross dimension is found in Table 1.

Table 1 Results of the peripheral cross task difficulty pilot experiment

\begin{tabular}{lll}
\hline $\begin{array}{l}\text { Cross dimensions } \\
\text { (pixels) }\end{array}$ & Aspect ratio & $\begin{array}{l}\text { Mean cross task } \\
\text { accuracy }(\%)\end{array}$ \\
\hline $104 \times 113$ (Exp. 1, 2) & 1.087 & $68.0 \%$ \\
$98 \times 120$ & 1.224 & $85.3 \%$ \\
$93 \times 127$ (Exp. 3) & 1.366 & $96.0 \%$ \\
$88 \times 134$ & 1.523 & $85.3 \%$ \\
\hline
\end{tabular}




\section{References}

Allen, R., \& McGeorge, P. (2008). Enumeration Shape information and expertise. Acta Psychologica, 129, 26-31.

Allen, R., \& McGeorge, P. (2011). Enumeration: Experts take their time. Applied Cognitive Psychology, 25, 588-592.

Ashkenazi, S., Mark-Zigdon, N., \& Avishai, H. (2013). Do subitizing deficits in developmental dyscalculia involve pattern recognition weakness? Developmental Science, 16, 35-46.

Bloechle, J., Huber, J. F., Klein, E., Bahnmueller, J., Rennig, J., Moeller, K., \& Huber, S. (2018). Spatial arrangement and set size influence the coding of non-symbolic quantities in the intraparietal sulcus. Frontiers in Human Neuroscience, 12, 54.

Briggs, G., Bridewell, W., \& Bello, P. F. (2017). A computational model of the role of attention in subitizing and enumeration. In: Proceedings of the 39th annual meeting of the cognitive science society (pp. 1672-1677). London, UK.

Burr, D. C., Turi, M., \& Anobile, G. (2010). Subitizing but not estimation of numerosity requires attentional resources. Journal of Vision, 10, 1-10.

Choo, H., \& Franconeri, S. (2014). Enumeration of small collections violates weber's law. Psychonomic Bulletin \& Review, 21, 93-99.

Coltheart, M. (1980). Iconic memory and visible persistence. Perception \& Psychophysics, 27, 183-228.

Egeth, H. E., Leonard, C. J., \& Palomares, M. (2008). The role of attention in subitizing: is the magical number 1? Visual Cognition, $16,463-473$.

Hyde, D. C., \& Wood, J. N. (2011). Spatial attention determines the nature of nonverbal number representation. Journal of Cognitive Neuroscience, 23, 2336-2351.

Jansen, B. R., Hofman, A. D., Straatemeier, M., Bers, B. M., Raijmakers, M. E., \& Maas, H. L. (2014). The role of pattern recognition in children's exact enumeration of small numbers. British Journal of Developmental Psychology, 32, 178-194.

Jevons, W. S. (1871). The power of numerical discrimination. Nature, 3, 281-282.

Jewell, S. W. (2007). Visual perception of shape and area in random dot patterns (Doctoral dissertation, Rice University). http://scholarship.rice.edu/handle/1911/20685.

Kaufman, E. L., Lord, M. W., Reese, T. W., \& Volkmann, J. (1949). The discrimination of visual number. The American Journal of Psychology, 62, 498-525.

Krajcsi, A., Szabó, E., \& Mórocz, I. Á.. (2013). Subitizing is sensitive to the arrangement of objects. Experimental Psychology, 60, 227234.

Logan, G. D., \& Zbrodoff, N. J. (2003). Subitizing and similarity: Toward a pattern-matching theory of enumeration. Psychonomic Bulletin \& Review, 10, 676-682.
Mandler, G., \& Shebo, B. J. (1982). Subitizing: an analysis of its component processes. Journal of Experimental Psychology: General, 111, 1-22.

Mazza, V., \& Caramazza, A. (2015). Multiple object individuation and subitizing in enumeration: a view from electrophysiology. Frontiers in Human Neuroscience, 9, 1-7.

Olivers, C. N., \& Watson, D. G. (2008). Subitizing requires attention. Visual Cognition, 16, 439-462.

Paolacci, G., Chandler, J., \& Ipeirotis, P. G. (2010). Running experiments on amazon mechanical turk. Judgment and Decision Making, 5, 411-419.

Peterson, S. A., \& Simon, T. J. (2000). Computational evidence for the subitizing phenomenon as an emergent property of the human cognitive architecture. Cognitive Science, 24, 93-122.

Piazza, M., Fumarola, A., Chinello, A., \& Melcher, D. (2011). Subitizing reflects visuo-spatial object individuation capacity. Cognition, 121, 147-153.

Railo, H., Koivisto, M., Revonsuo, A., \& Hannula, M. M. (2008). The role of attention in subitizing. Cognition, 107, 82-104.

Rensink, R. A. (2000). The dynamic representation of scenes. Visual Cognition, 7, 17-42.

Sathian, K., Simon, T. J., Peterson, S., Patel, G. A., Hoffman, J. M. \& Grafton, S. T. (1999). Neural evidence linking visual object enumeration and attention. Journal of Cognitive Neuroscience, 11, $36-51$.

Treisman, A. M., \& Gelade, G. (1980). A feature-integration theory of attention. Cognitive Psychology, 12, 97-136.

Trick, L. M. (1992). A theory of enumeration that grows out of a general theory of vision: subitizing, counting, and finsts. Advances in Psychology, 91, 257-299.

Trick, L. M., \& Enns, J. T. (1997). Clusters precede shapes in perceptual organization. Psychological Science, 8, 124-129.

Trick, L. M., \& Pylyshyn, Z. W. (1994). Why are small and large numbers enumerated differently? a limited-capacity preattentive stage in vision. Psychological Review, 101, 80-102.

Vetter, P., Butterworth, B., \& Bahrami, B. (2008). Modulating attentional load affects numerosity estimation: Evidence against a pre-attentive subitizing mechanism. PloS One, 3, 1-6.

Wender, K. F., \& Rothkegel, R. (2000). Subitizing and its subprocesses. Psychological Research Psychologische Forschung, 64, 81-92.

Whalen, J., Gallistel, C. R., \& Gelman, R. (1999). Nonverbal counting in humans: The psychophysics of number representation. Psychological Science, 10, 130-137.

Wolters, G., Van Kempen, H., \& Wijlhuizen, G.-J. (1987). Quantification of small numbers of dots: Subitizing or pattern recognition? The American Journal of Psychology, 100, 225-237.

Publisher's note Springer Nature remains neutral with regard to jurisdictional claims in published maps and institutional affiliations. 